\title{
December 2017 Pulmonary Case of the Month
}

\section{Lewis J. Wesselius, $M D^{1}$ \\ Michael B. Gotway, MD²}

\author{
Departments of ${ }^{1}$ Pulmonary Medicine and ${ }^{2}$ Radiology \\ Mayo Clinic Arizona \\ Scottsdale, AZ USA
}

\section{History of Present IIIness}

A 52-year-old woman from lowa sought a second opinion for a left hilar mass. She travels to Phoenix regularly to visit family. She began feeling ill in late 2016 with cough and sputum production and was treated with multiple courses of antibiotics without improvement.

\section{$\mathrm{PMH}, \mathrm{SH}$ and $\mathrm{FH}$}

Past medical history is unremarkable. She is a nonsmoker. FH is noncontributory.

\section{Physical Examination}

Physical examination was normal.

\section{Radiography}

In March of this year she had chest radiograph in Phoenix which suggested left hilar adenopathy. A thoracic CT scan was performed (Figure 1).

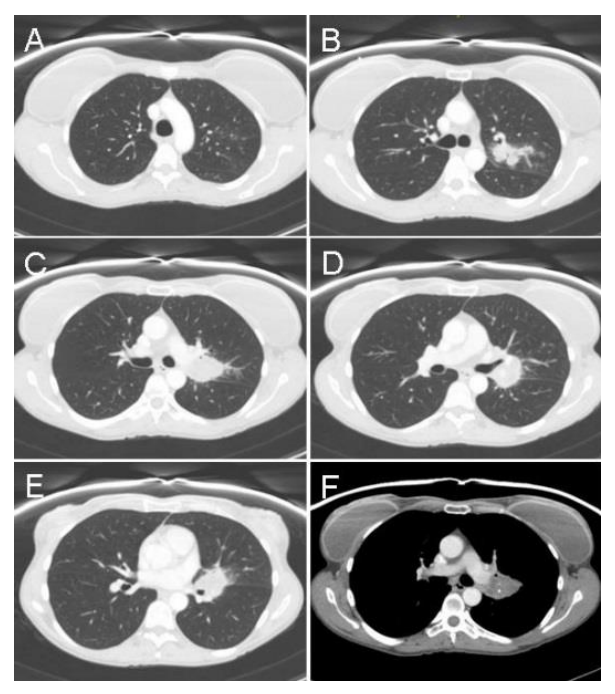

Figure 1. Representative images from the thoracic CT scan in lung windows (A-E) and soft tissue windows $(F)$. 
Which of the following are diagnostic considerations?

1. Lung cancer

2. Lymphoma

3. Sarcoidosis

4. Tuberculosis

5. All of the above 


\section{Correct! \\ 5. All of the above}

The thoracic CT scan shows left hilar enlargement secondary to a hilar mass. The mass appears to be unilateral. The differential diagnosis of a hilar mass is large but includes malignant causes such as lung cancer or lymphoma or benign causes such as tuberculosis, fungal disease or sarcoidosis. An incidental finding is that the patient has had bilateral augmentation mammoplasties.

Serologic testing for coccidioidomycosis and histoplasmosis were negative and a serum angiotensin converting enzyme level was normal. She returned to lowa in May but continued to have cough and chest heaviness. A repeat thoracic CT scan was performed (Figure 2).

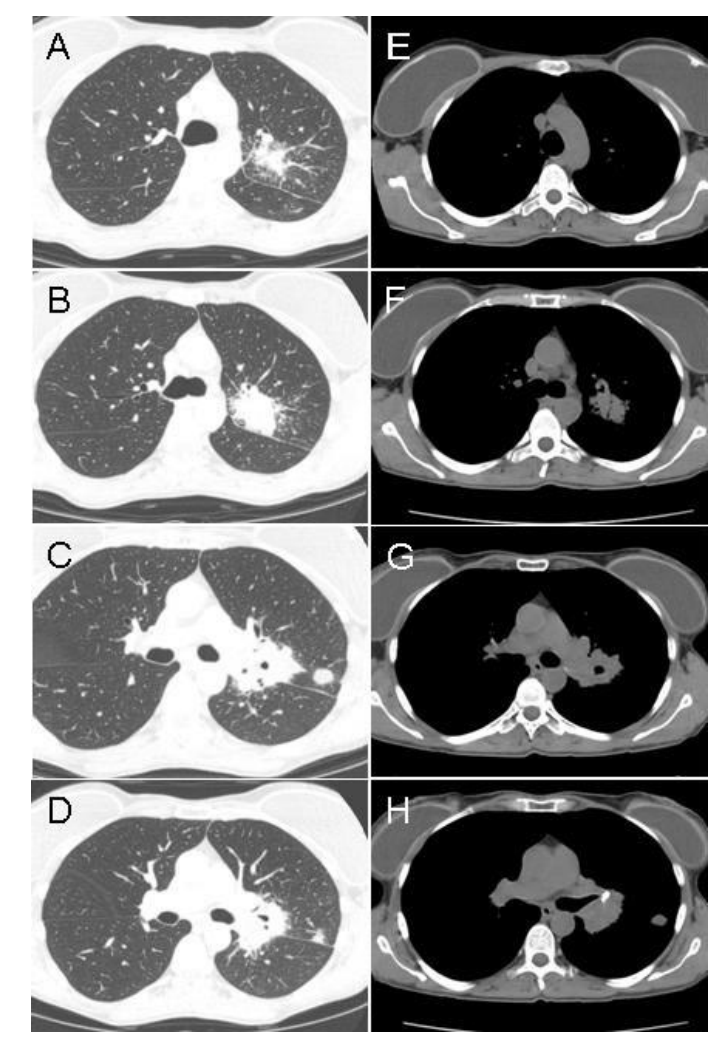

Figure 2. Repeat CT scan in lung windows (A-D) and soft tissue windows $(E-H)$.

Which of the following is/are true?

1. The calcium present in the left hilar region excludes a malignant cause

2. The lack of bilateral hilar enlargement excludes sarcoidosis for all practical purposes

3. There is now definite lung involvement on the left

4. 1 and 3

5. All of the above 


\section{Correct!}

\section{There is now definite lung involvement on the left}

The calcium present in the left hilar region is small, eccentric and likely old. While eccentric calcification within a nodule suggests the nodule is more likely benign than malignant, eccentric (and stippled) patterns of calcification within pulmonary nodules can be seen in malignancies. The presence of bilateral hilar enlargement might make sarcoidosis more likely but unilateral enlargement would not exclude the diagnosis. There is now definite lung involvement on the left which is new compared to the previous CT scan.

Which of the following are indicated at this time?

1. Bronchoscopy with endobronchial ultrasound (EBUS)

2. Continued observation

3. Needle biopsy

4. PET/CT scan

5. Video assisted thorascopic biopsy (VATS) 


\section{Correct!}

\section{Bronchoscopy with endobronchial ultrasound (EBUS)}

The presence of the new lung lesion is worrisome and suggests progression of her disease. It seems unlikely that a PET/CT scan would be useful since most of the diagnostic possibilities would likely result in increased uptake in the lung and the hilar mass. Regardless, it seems that a tissue diagnosis is warranted and either bronchoscopy, needle biopsy or VATS seem reasonable. Her physicians in lowa chose to proceed with bronchoscopy with EBUS. The biopsy from the enlarged left hilar lymph nodes showed lymphocytes and inflammation but no malignancy. Special stains and cultures were negative. They felt the diagnosis was unclear but most likely sarcoidosis.

She returned to Phoenix in June. Her symptoms were unchanged. Another thoracic CT was very similar to the one done in lowa.

Which of the following is indicated at this time?

1. Chamberlin procedure for biopsy of the left hilar lymph node

2. Needle biopsy of the lung lesion

3. Repeat bronchoscopy with EBUS

4. Video-assisted thorascopic surgery (VATS)

5. None of the above 


\section{Correct! \\ 3. Repeat bronchoscopy with EBUS}

She still needs a tissue diagnosis and repeat bronchoscopy with EBUS, needle biopsy of the lung lesion or VATS seems reasonable. A Chamberlain procedure is an anterior mediastinal lymph node exploration which allows access to station 5 and 6 lymph nodes via the left second intercostal space. It has largely been supplanted by bronchoscopy with EBUS. We chose to repeat the bronchoscopy with EBUS. It revealed a narrowed LUL bronchus (Figure 3).

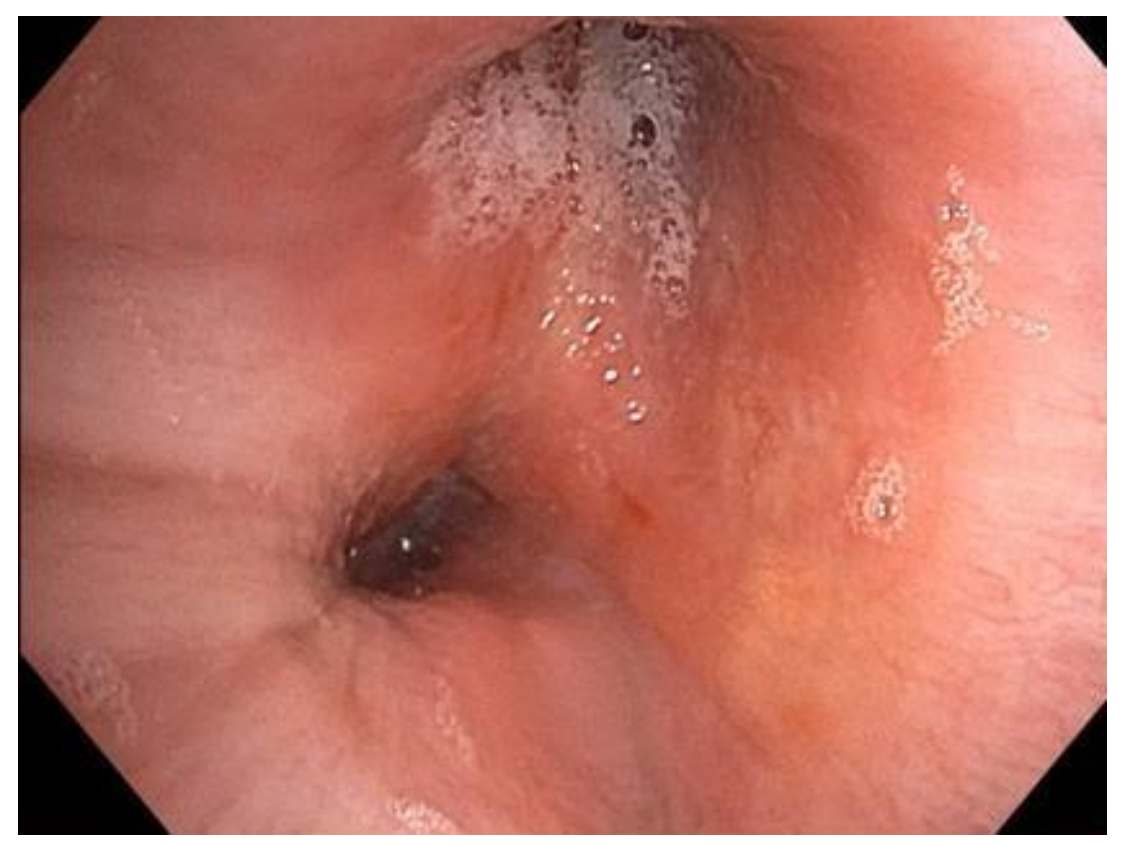

Figure 3. Photograph of the left upper lobe bronchus showing narrowing and splaying of the carina.

Biopsies were taken with EBUS and transbronchial biopsies also performed. The EBUS revealed lymphocytes with a possible germinal center. No malignancy or granulomas were seen. The transbronchial biopsies showed a mixed inflammatory infiltrate with lymphocytes, plasma cells, histiocytes, eosinophils and small amount of necrotic debris. No malignancy was identified and smears and cultures were also negative.

\section{What should be done at this time?}

1. Needle biopsy

2. Repeat bronchoscopy with EBUS

3. VATS

4. 1 or 3

5. Any of the above 


\section{Correct! \\ 1. Needle biopsy}

\section{VATS}

4. 1 or 3

Really any are reasonable, although having failed twice before, repeating a bronchoscopy with EBUS for a third time seems unlikely to result in a diagnosis. A needle biopsy with CT guidance was performed. The histology showed acute inflammation with necrosis and granulation tissue (Figure 4).
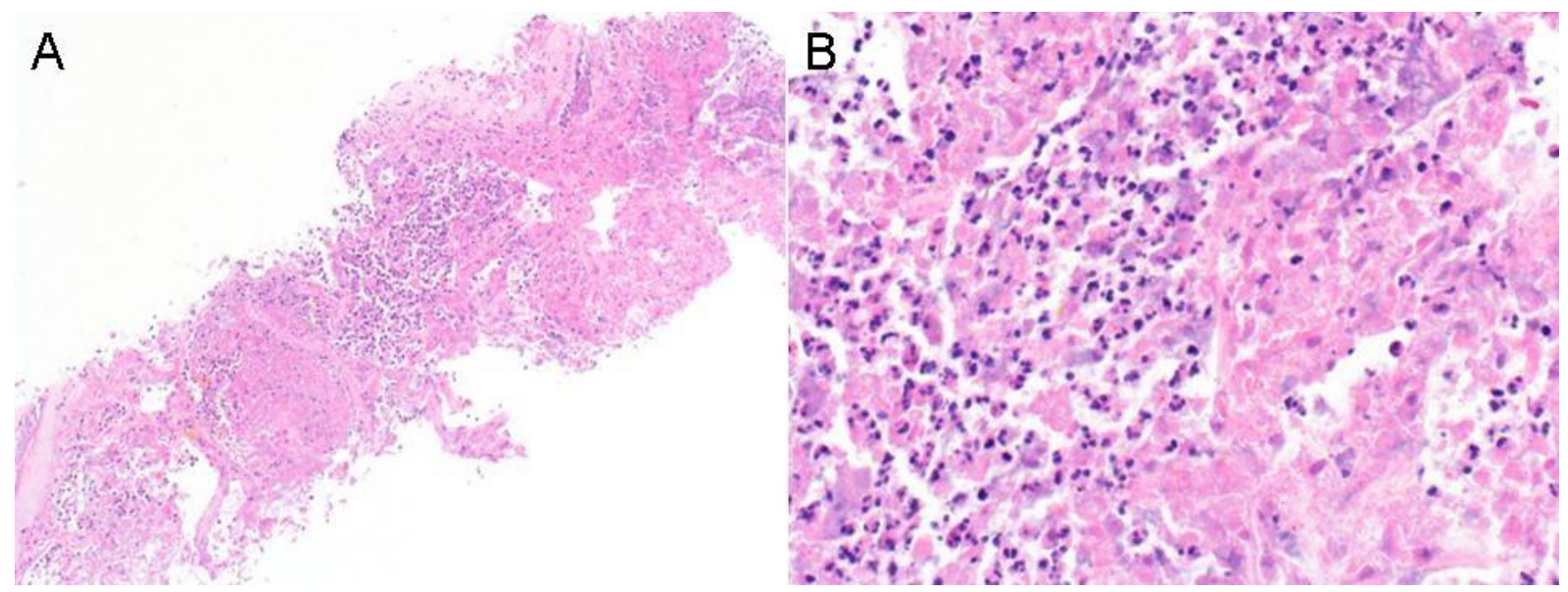

Figure 3. Needle biopsy of the lung. A: H\&E staining low power. B: H\&E staining high power.

No malignancy was identified and acid fast bacilli and Grocott's methenamine silver stain (GMS) were negative.

Because a definitive diagnosis was not made, the patient was referred for VATS biopsy. The histology again was nondiagnostic showing necrotizing and cavitating granulomatous inflammation surrounded by hyalinizing fibrosis (Figure 5).

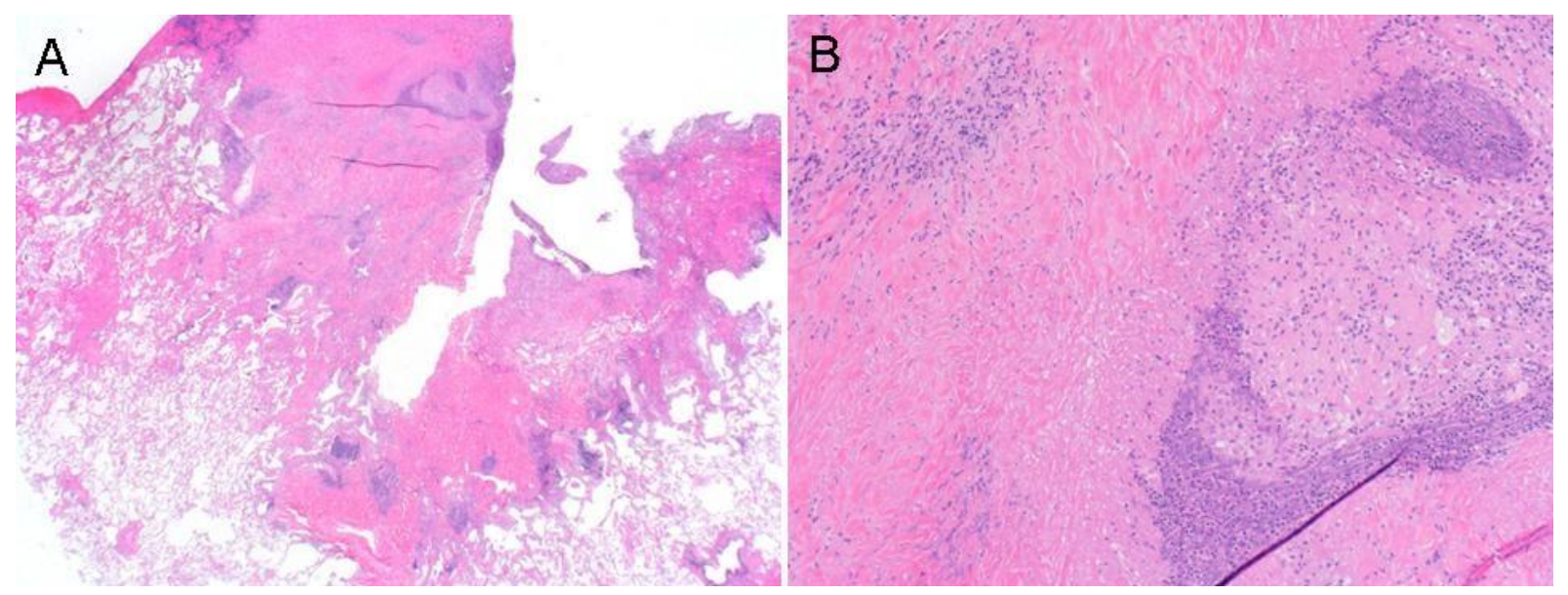


Figure 5. VATS biopsy of the lung. A: H\&E staining low power. B: H\&E staining high power.

At about this time a complement fixation test for histoplasmosis yeast returned positive at 1:16. The complement fixation against histoplasmosis mycelia and a histoplasmosis antibody test were negative.

What should be done next?

1. Continued observation

2. Treat with corticosteroids

3. Treat with itraconazole

4. 1 or 3

5. Any of the above 


\section{Correct! \\ 3. Treat with itraconazole}

We were uncertain if the clinical picture represented active histoplasmosis (1). About a third of patients with active histoplasmosis have a complement fixation titer for yeast of only $1: 8$ or $1: 16$. We thought that the patient most likely had smoldering histoplasmosis and was developing fibrotic scarring in her perihilar region. This would be similar to fibrosing mediastinitis from histoplasmosis (2). We were reluctant to treat with corticosteroids given her positive histoplasmosis complement fixation test, and therefore, treated her with itraconazole. Her cough quickly improved and a repeat thoracic CT shows the hilar mass and the lung lesions to be smaller in size. There are plans for close clinical and radiologic follow up.

\section{References}

1. Wheat LJ, Azar MM, Bahr NC, Spec A, Relich RF, Hage C. Histoplasmosis. Infect Dis Clin North Am. 2016 Mar;30(1):207-27. [CrossRef] [PubMed]

2. Koksal $\mathrm{D}$, Bayiz $\mathrm{H}$, Mutluay $\mathrm{N}$, et al. Fibrosing mediastinitis mimicking bronchogenic carcinoma. J Thorac Dis. 2013 Feb;5(1):E5-7. [CrossRef] [PubMed] 